\title{
Exploring tidal effects of coalescing binary neutron stars in numerical relativity II: Longterm simulations
}

\author{
Kenta Hotokezaka, ${ }^{1}$ Koutarou Kyutoku, ${ }^{2}$ Hirotada Okawa,,${ }^{3,4}$ and Masaru Shibata ${ }^{3}$ \\ ${ }^{1}$ Racah Institute of Physics, The Hebrew University of Jerusalem, Jerusalem, 91904, Israel \\ ${ }^{2}$ Department of Physics, University of Wisconsin-Milwaukee, \\ P.O. Box 413, Milwaukee, Wisconsin 53201, USA \\ ${ }^{3}$ Yukawa Institute for Theoretical Physics, Kyoto University, Kyoto, 606-8502, Japan \\ ${ }^{4}$ Advanced Research Institute for Science 86 Engineering, \\ Waseda University, 3-4-1 Okubo, Shinjuku, Tokyo 169-8555, Japan
}

(Dated: July 12, 2018)

\begin{abstract}
We perform new longterm (15-16 orbits) simulations of coalescing binary neutron stars in numerical relativity using an updated Einstein's equation solver, employing low-eccentricity initial data, and modeling the neutron stars by a piecewise polytropic equation of state. A convergence study shows that our new results converge more rapidly than the third order and using the determined convergence order, we construct an extrapolated waveform for which the estimated total phase error should be less than 1 radian. We then compare the extrapolated waveforms with those calculated by the latest effective-one-body (EOB) formalism in which the so-called tidal deformability, higher post-Newtonian corrections, and gravitational self-force effects are taken into account. We show that for a binary of compact neutron stars with their radius $11.1 \mathrm{~km}$, the waveform by the EOB formalism agrees quite well with the numerical waveform so that the total phase error is smaller than 1 radian for the total phase of $\sim 200$ radian up to the merger. By contrast, for a binary of less compact neutron stars with their radius $13.6 \mathrm{~km}$, the EOB and numerical waveforms disagree with each other in the last few wave cycles, resulting in the total phase error of $\sim 3$ radian.
\end{abstract}

PACS numbers: 04.25.D-, 04.30.-w, 04.40.Dg

\section{INTRODUCTION}

The inspiral and merger of coalescing compact binaries are among the most promising sources for groundbased kilometer-size laser-interferometric gravitationalwave detectors [1]. A statistical study based on the stellar-evolution synthesis (e.g., Refs. [2, 3] ) suggests that the detection rate for them will be $\sim 1-100 \mathrm{yr}^{-1}$ for advanced detectors, i.e., advanced LIGO [4], advanced VIRGO [5], and KAGRA [6], which will sequentially start operation in the coming years.

One of the important steps after the first detection of gravitational waves from binary neutron stars (and also a black hole-neutron star binary) will be to extract binary parameters such as mass, spin, and radius of each object in the binary systems. In particular, the mass and radius (or a quantity related to it) of the neutron stars have invaluable information for determining the equation of state (EOS) of the neutron-star matter, which is still poorly known. The mass of two neutron stars will be determined with a high accuracy $\lesssim 1 \%$, if the gravitationalwave signals in the inspiral stage are detected with the signal-to-noise ratio $\gtrsim 10$ and the neutron-star spins are supposed to be negligible [7]. On the other hand, determining the parameters related to the neutron-star radius is the challenging issue although it has to be done for constraining the neutron-star EOS (e.g., Ref. 8- 10]). Among other possible methods, extracting the tidal deformability of the neutron stars from gravitational waves emitted binary-neutron-star inspirals is one of the most promising methods 11 13. For employing this method, we have to prepare a theoretical template of gravitational waves from binary-neutron-star inspirals taking into account tidal-deformation effects that influence the dynamics of the late inspiral orbits (e.g., Ref. [14]). Hence, theoretically deriving a precise gravitational waveform for binary-neutron-star inspirals including the tidal effects is an urgent task.

A post-Newtonian ( $\mathrm{PN})$ gravitational waveform for the early stage of binary-neutron-star inspirals (with the frequency $f \lesssim 400 \mathrm{~Hz}$ ) was first derived by Flanagan and Hinderer including the leading-order tidal effects [12]. They showed that the tidal effect for the evolution of the gravitational-wave phase could be described only by the tidal deformability of neutron stars. They also found that the tidal deformability of neutron stars could be measured by the advanced gravitational-wave detectors by using the gravitational-wave signals for $f=10-400 \mathrm{~Hz}$, if the tidal deformability of neutron stars is sufficiently large or if we could observe an event with a high signalto-noise ratio (see also Ref. 15]). If the waveform is extended to the higher frequency range, the measurability can be significantly improved. In the PN approach, however, the uncertainty of the higher-order PN terms prevents us to construct the accurate waveform at the higher frequency [16 18].

To overcome the ambiguity in the higher PN terms, an effective one body (EOB) formalism with the tidal effects has been explored [13, 19, 20]. In this approach, the nontidal part is calibrated using the results of binary-blackhole simulations. Damour and his collaborators 21] subsequently explored the measurability of the tidal de- 
formability with the advanced gravitational-wave detectors employing an EOB formalism including tidal effects up to the second PN order. They concluded that the tidal deformability of neutron stars could be measured by the advanced gravitational-wave detectors if the signalto-noise ratio of the gravitational-wave signal is higher than $\sim 16$ for any EOS that satisfies the constraint of the maximum gravitational mass $\gtrsim 2 M_{\odot}$ [22]. The key assumption of their study is that the EOB approach is valid up to the onset of the merger of binary neutron stars. However, in the stage just before the merger, effects such as nonlinear tidal-deformation effects, which are not taken into account in the current EOB formalism, could come into play (see, e.g., Ref. [23]).

For precisely understanding the orbital motion and the waveform in the late inspiral stage of binary neutron stars, a high-resolution numerical-relativity (NR) simulation with appropriately physical setting is obviously necessary. Recently, long-term simulations for binary-neutron-star inspirals were performed by three groups [20, 23 28] aiming at the derivation of accurate gravitational waveforms for the late inspiral stage. They followed the late binary inspiral for $\lesssim 10$ orbits up to the onset of the merger. However, in their numerical simulations, an unphysical residual eccentricity is present in the initial data. This seriously made their results less accurate, because binary neutron stars in the late inspiral stage are believed to have a quasi-circular orbit with negligible eccentricity. In the present work, we simulate binary-neutron-star inspirals for a longer term with more physical initial data in which the eccentricity is sufficiently small (less than $\left.10^{-3}\right)^{1}$. In addition, we perform the simulations employing a formalism in which the constraint violation can be suppressed to a level much smaller than that in our previous study [23]. As a result, we can obtain an extrapolated waveform in a much more accurate and reliable manner than in our previous study.

The paper is organized as follows. In Sec. II, we summarize the formulation and numerical schemes employed in our numerical-relativity study, and also review the EOS employed. In Sec. III, we describe our method for deriving an extrapolated gravitational waveform, showing the resulting waveforms that are much more accurate than those derived in our previous study 23. We then compare our extrapolated waveforms with those derived by the latest EOB approach and examine its accuracy in Sec. IV. Section V is devoted to a summary. Throughout this paper, we employ the geometrical units of $c=G=1$ where $c$ and $G$ are the speed of light and the gravitational constant, respectively.

\footnotetext{
${ }^{1}$ We note that R. Haas and his collaborators (SXS collaboration) have also derived the waveforms of small eccentricity in their longterm simulations, although their results have not been published yet.
}

\section{FORMULATION FOR NUMERICAL-RELATIVITY SIMULATION}

In this section, we briefly describe the formulation and the numerical schemes of our numerical-relativity simulation employed in this work.

\section{A. Evolution and Initial Condition}

We follow the inspiral and early stage of the merger of binary neutron stars using our numerical-relativity code, SACRA, for which the details are described in Ref. 29]. In this work, we employ a moving puncture version of the Baumgarte-Shapiro-Shibata-Nakamura formalism [30], locally incorporating a Z4c-type constraint propagation prescription [31] (see 32] for our implementation) for a solution of Einstein's equation. The constraint propagation from the neutron-star's outer region plays a crucial role for reducing the constraint violation and for improving the order of the convergence as discovered in Ref. 31]. In our numerical simulation, a fourth-order finite differencing scheme in space and time is used implementing an adaptive mesh refinement (AMR) algorithm. At refinement boundaries, a secondorder interpolation scheme is partly used. The advection terms are evaluated by fourth-order lop-sided upwindtype finite differencing [33]. A fourth-order Runge-Kutta method is employed for the time evolution. For the hydrodynamics, a high-resolution central scheme based on a Kurganov-Tadmor scheme [34] with a third-order piecewise parabolic interpolation and with a steep min-mod limiter is employed.

In this work, we prepare nine refinement levels for the AMR computational domain. Specifically, two sets of four finer domains comoving with each neutron star cover the region of their vicinity. The other five coarser domains cover both neutron stars by a wider domain with their origins fixed at the center of the mass of the binary system. Each refinement domain consists of a uniform, vertex-centered Cartesian grid with $(2 N+1,2 N+1, N+$ 1 ) grid points for $(x, y, z)$ (the equatorial plane symmetry at $z=0$ is imposed). The half of the edge length of the largest domain (i.e., the distance from the origin to outer boundaries along each axis) is denoted by $L$, which is chosen to be larger than $\lambda_{0}$, where $\lambda_{0}=\pi / \Omega_{0}$ is the initial wavelength of gravitational waves and $\Omega_{0}$ is the initial orbital angular velocity. The grid spacing for each domain is $\Delta x_{l}=L /\left(2^{l} N\right)$, where $l=0-8$. In this work, we choose $N=72,60,48$, and 40 for examining the convergence properties of numerical results. With the highest grid resolution (for $N=72$ ), the semimajor diameter of each neutron star is covered by about 120 grid points.

We prepare binary neutron stars in quasi-circular orbits for the initial condition of numerical simulations. These initial conditions are numerically obtained by using a spectral-method library, LORENE [35]. We fol- 
TABLE I: Equations of state (EOS) employed, the radius and the tidal Love number of $l=(2,3,4)$ of spherical neutron stars of mass $1.35 M_{\odot}$, the radius of light ring orbit, angular velocity of initial data, and the finest grid spacing in the four different resolution runs. $m_{0}$ denotes the total mass of the system. In this study, it is $2.7 M_{\odot}$.

\begin{tabular}{cccccccc}
\hline \hline EOS & $R_{1.35}(\mathrm{~km})$ & $k_{2,1.35}$ & $k_{3,1.35}$ & $k_{4,1.35}$ & $r_{\text {LR }}$ & $m_{0} \Omega_{0}$ & $\Delta x_{8}(\mathrm{~km})$ \\
\hline APR4 & 11.1 & 0.0908 & 0.0234 & 0.00884 & 3.61 & 0.0156 & $0.140,0.167,0.209,0.251$ \\
H4 & 13.6 & 0.115 & 0.0326 & 0.0133 & 4.21 & 0.0155 & $0.183,0.220,0.274,0.329$ \\
\hline \hline
\end{tabular}

low $15-16$ orbits in this study. To do so, the orbital angular velocity of the initial configuration is chosen to be $m_{0} \Omega_{0} \approx 0.0155$ ( $f \approx 370 \mathrm{~Hz}$ for the total mass $m_{0}=2.7 M_{\odot}$, i.e., each mass of neutron stars is $\left.1.35 M_{\odot}\right)$. The neutron stars are assumed to have an irrotational velocity field, which is believed to be an astrophysically realistic configuration [36, 37]. The parameters for the initial models are listed in Table 1 .

For the computation of an accurate gravitational waveform in numerical simulations, we have to employ initial data of a quasi-circular orbit of negligible eccentricity. Namely, the eccentricity of the initial binary orbit has to be reduced to be as small as possible. Such initial data are constructed by an eccentricity-reduction procedure described in [32]. For the initial data employed in this work, the residual eccentricity is $\lesssim 10^{-3}$.

\section{B. Equation of State}

Following previous works [23, 32], we employ a parameterized piecewise-polytropic equation of state proposed by Read and her collaborators [38]. This EOS is written in terms of four segments of polytropes

$$
P=K_{i} \rho^{\Gamma_{i}} \quad\left(\text { for } \rho_{i} \leq \rho<\rho_{i+1}, 0 \leq i \leq 3\right)
$$

where $\rho$ is the rest-mass density, $P$ is the pressure, $K_{i}$ is a polytropic constant, and $\Gamma_{i}$ is an adiabatic index. At each boundary of the piecewise polytropes, $\rho=\rho_{i}$, the pressure is required to be continuous, i.e., $K_{i} \rho_{i+1}^{\Gamma_{i}}=K_{i+1} \rho_{i+1}^{\Gamma_{i+1}}$. Following Read and her collaborators, these parameters are determined in the following manner 38]: The crust EOS is fixed by setting $\Gamma_{0}=1.3562395$ and $K_{0}=3.594 \times 10^{13}$ in cgs units. The values of the boundary density is set as $\rho_{2}=10^{14.7} \mathrm{~g} / \mathrm{cm}^{3}$ and $\rho_{3}=10^{15.0} \mathrm{~g} / \mathrm{cm}^{3}$. With this preparation, the following four parameters become free parameters that should be given: $\left\{P_{1}, \Gamma_{1}, \Gamma_{2}, \Gamma_{3}\right\}$. Here, $P_{1}$ is the pressure at $\rho=\rho_{2}$, and for a given value of this, $K_{1}$ and $K_{i}$ are determined by $K_{1}=P_{1} / \rho_{2}^{\Gamma_{1}}$ and $K_{i+1}=K_{i} \rho_{i+1}^{\Gamma_{i}-\Gamma_{i+1}}$. In this work, we choose two sets of piecewise-polytropic EOS mimicking APR4 [39] and H4 [40] EOS (see Table 1 of Ref. [41] for the four parameters).

In numerical simulations, we employ a modified version of the piecewise polytropic EOS to approximately take into account thermal effects, which play a role in the merger phase. In this EOS, we decompose the pressure and specific internal energy into the cold and thermal parts as

$$
P=P_{\text {cold }}(\rho)+P_{\text {th }}, \quad \varepsilon=\varepsilon_{\text {cold }}(\rho)+\varepsilon_{\text {th }} .
$$

The cold parts of both variables are calculated using the original piecewise polytropic $\operatorname{EOS}$ from $\rho$, and then the thermal part of the specific internal energy is defined from $\varepsilon$ as $\varepsilon_{\text {th }}=\varepsilon-\varepsilon_{\text {cold }}(\rho)$. Because $\varepsilon_{\text {th }}$ vanishes in the absence of shock heating, it is regarded as the finitetemperature part determined by the shock heating in the present context. For the thermal pressure, a $\Gamma$-law idealgas EOS was adopted as

$$
P_{\mathrm{th}}=\left(\Gamma_{\mathrm{th}}-1\right) \rho \varepsilon_{\mathrm{th}} .
$$

Following our latest works [32, 41], $\Gamma_{\text {th }}$ is chosen to be 1.8 .

\section{Extraction of Gravitational waves}

Gravitational waves are extracted from the outgoingcomponent of complex Weyl scalar $\Psi_{4}$ [29]. From this, gravitational waveforms are determined in spherical coordinates $(r, \theta, \phi)$ by

$$
h:=h_{+}(t)-i h_{\times}(t)=-\lim _{r \rightarrow \infty} \int^{t} d t^{\prime} \int^{t^{\prime}} d t^{\prime \prime} \Psi_{4}\left(t^{\prime \prime}, r\right) .
$$

Here, we omit arguments $\theta$ and $\phi . \Psi_{4}$ can be expanded in the form

$$
\Psi_{4}(t, r, \theta, \phi)=\sum_{l m} \Psi_{4}^{l, m}(t, r)_{-2} Y_{l m}(\theta, \phi),
$$

where ${ }_{-2} Y_{l m}$ denotes the spin-weighted spherical harmonics of weight -2 and $\Psi_{4}^{l, m}$ are expansion coefficients defined by this equation. In this work, we focus only on the $(l,|m|)=(2,2)$ mode because we pay attention only to the equal-mass binary, and hence, this quadrupole mode is the dominant one.

We evaluate $\Psi_{4}$ at a finite spherical-coordinate radius $r / m_{0}=100-240$. To compare the waveforms extracted at different radii, we use the retarded time defined by

$$
t_{\text {ret }}:=t-r_{*},
$$

where $r_{*}$ is the so-called tortoise coordinate defined by

$$
r_{*}:=r_{\mathrm{A}}+2 m_{0} \ln \left(\frac{r_{\mathrm{A}}}{2 m_{0}}-1\right),
$$

with $r_{\mathrm{A}}:=\sqrt{A / 4 \pi}$ and $A$ the proper area of the extraction sphere. 


\section{RECIPE FOR CONSTRUCTING AN EXTRAPOLATED WAVEFORM}

In this section, we present our prescription for deriving an extrapolated gravitational waveform from raw numerical data of $\Psi_{4}$, and show that the resulting waveforms have a good accuracy that can be compared carefully with the EOB results.

\section{A. Extrapolation to infinite extraction radius}

As we mentioned in the previous section, we extract $\Psi_{4}$ at several coordinate radii, $100-240 m_{0}$, and then, this complex Weyl scalar is decomposed into the spherical harmonics components, $\Psi_{4}^{l, m}$. Since the waveform of $\Psi_{4}$ extracted at a finite radius, $r_{0}$, is systematically different from that at null infinity, we first compute an extrapolated waveform at $r_{0} \rightarrow \infty$ using the Nakano's method as 42,43 .

$$
\begin{aligned}
\Psi_{4}^{l, m, \infty}\left(t_{\mathrm{ret}}, r_{0}\right)= & C\left(r_{0}\right)\left[\Psi_{4}^{l, m}\left(t_{\mathrm{ret}}, r_{0}\right)\right. \\
& \left.-\frac{(l-1)(l+2)}{2 r_{\mathrm{A}}} \int^{t_{\mathrm{ret}}} \Psi_{4}^{l, m}\left(t^{\prime}, r_{0}\right) d t^{\prime}\right]
\end{aligned}
$$

where $C\left(r_{0}\right)$ is a function of $r_{0}$. Since our coordinates are similar to isotropic coordinates of non-rotating black holes, we choose $r_{\mathrm{A}}=r_{0}\left[1+m_{0} /\left(2 r_{0}\right)\right]^{2}$. $C\left(r_{0}\right)$ depends on the choice of the tetrad components; for our choice, it is appropriate to choose $C\left(r_{0}\right)=1-2 m_{0} / r_{\mathrm{A}}$. In this setting, $t_{\text {ret }}$ at $r=r_{0}$ is given by Eqs. (2.6) and (2.7).

The left panel of Fig. 1 plots the real part of $\Psi_{4}^{2,2, \infty}\left(t_{\text {ret }}, r_{0}\right)$ for several choices of $r_{0}$. The right panel shows the evolution of the absolute amplitude of $\Psi_{4}^{2,2, \infty}\left(t_{\text {ret }}, r_{0}\right)$. These show that the extrapolated waveforms depend very weakly on the extraction radius, $r_{0}$ (see Ref. 43] for the reason).

We then have to calibrate how weakly the resulting extrapolated waveforms, $\Psi_{4}^{2,2, \infty}\left(t_{\text {ret }}, r_{0}\right)$, depend on $r_{0}$ and have to estimate the systematic error in this quantity. We find that the systematic error in phase decreases approximately in proportional to $r_{0}^{-1}$ (cf. the left lower panel of Fig. 1 that indeed shows this property). Figure 1 implies that for $r_{0} \gtrsim 200 m_{0}$, the systematic error in phase is smaller than 0.3 radian. This value is smaller than the error in the extrapolated waveform finally obtained (associated with the uncertainty in the resolution extrapolation), and can be accepted in the present numerical study. Note that this phase error is systematic and could be subtracted, although we do not do so in this work.

By contrast, the systematic error in amplitude is appreciable, i.e., $1-2$ percents even for $r_{0} \approx 200 m_{0}$. For suppressing this error, we might have to enlarge the computational domain for the wave extraction. However, this error size is smaller than another error associated with the spurious short-term modulation in the numerical gravitational-wave amplitude as reported in Ref. [32]: The right panel of Fig. 1] shows that a modulation in the amplitude is present with its fluctuation amplitude of $\lesssim 3 \%$ in particular in the early stages of the numerical waveform. Since this error was not able to be cleaned up, we do not take a further extrapolation of $\left|\Psi_{4}^{l, m, \infty}\left(t_{\text {ret }}, r_{0}\right)\right|$ for $r_{0} \rightarrow \infty$. Thus, in this work, we employ $\Psi_{4}^{l, m, \infty}\left(t_{\text {ret }}, r_{0}\right)$ computed from the data extracted at $r_{0}=200 m_{0}$ [hereafter written as $\left.\Psi_{4}^{l, m, \infty}\left(t_{\mathrm{ret}}\right)\right]$ without further processing and perform subsequent analyses keeping in mind that in the amplitude extrapolated by Eq. (3.1), there could exist a local error in magnitude up to $\sim 3 \%$ of the exact amplitude (note that in average the error would be much smaller than $3 \%$ ).

\section{B. Extrapolation for zero-grid spacing limit}

Next, we consider the resolution extrapolation for the limit $\Delta x_{8} \rightarrow 0$. For this task, numerical simulations have to be performed for more than three grid resolutions. In this study, we performed four simulations for each model employing four different grid resolutions (cf. Table \ for the finest grid spacing, $\Delta x_{8}$, for each run). For each run, we extracted the numerical waveform at $r_{0}=200 m_{0}$ and then performed the extrapolation of $r_{0} \rightarrow \infty$ as described in Eq. (3.1).

We then need to perform an extrapolation procedure of taking the zero grid-spacing limit for obtaining an approximately exact solution. For this procedure, we first analyze the relation of the time to the merger, $t_{\mathrm{mrg}}$, as a function of $\Delta x_{8}$ following Ref. [23]. Here, the merger time, $t_{\mathrm{mrg}}$, is defined as the time at which the maximum value of $\left|\Psi_{4}^{2,2, \infty}\left(t_{\text {ret }}\right)\right|$ is recorded. Then, it is found that $t_{\mathrm{mrg}}$ converges to an unknown exact value at $\sim 4$ th order (see below for more detailed analysis). $t_{\mathrm{mrg}}$ is larger for the better grid resolutions because for the lower grid resolutions, the numerical dissipation is larger and the inspiraling process is spuriously accelerated. This numerical error is universally present for finite values of $\Delta x_{8}$; namely, for any inspiraling stage in any numerical simulations, the error is always present. For obtaining the "exact" waveform, thus, we always need an extrapolation procedure. Then, the next question is how to extrapolate the waveform for the limit $\Delta x_{8} \rightarrow 0$. We propose the following method in this study.

We first determine the gravitational waveform and time evolution of the angular frequency as functions of $t_{\text {ret }}$ by integrating $\Psi_{4}^{l, m, \infty}\left(t_{\text {ret }}\right)$ for each raw numerical data. Here, the gravitational waveform for each multipole mode satisfies [see Eq. (2.4)]

$$
\ddot{h}^{l, m}:=\ddot{h}_{+}^{l, m}-i \ddot{h}_{\times}^{l, m}=-\Psi_{4}^{l, m, \infty}\left(t_{\mathrm{ret}}\right) .
$$

$h^{l, m}$ is obtained by the double time integration of $\Psi_{4}^{l, m, \infty}$. For this procedure, we employ the method of Ref. [44], 

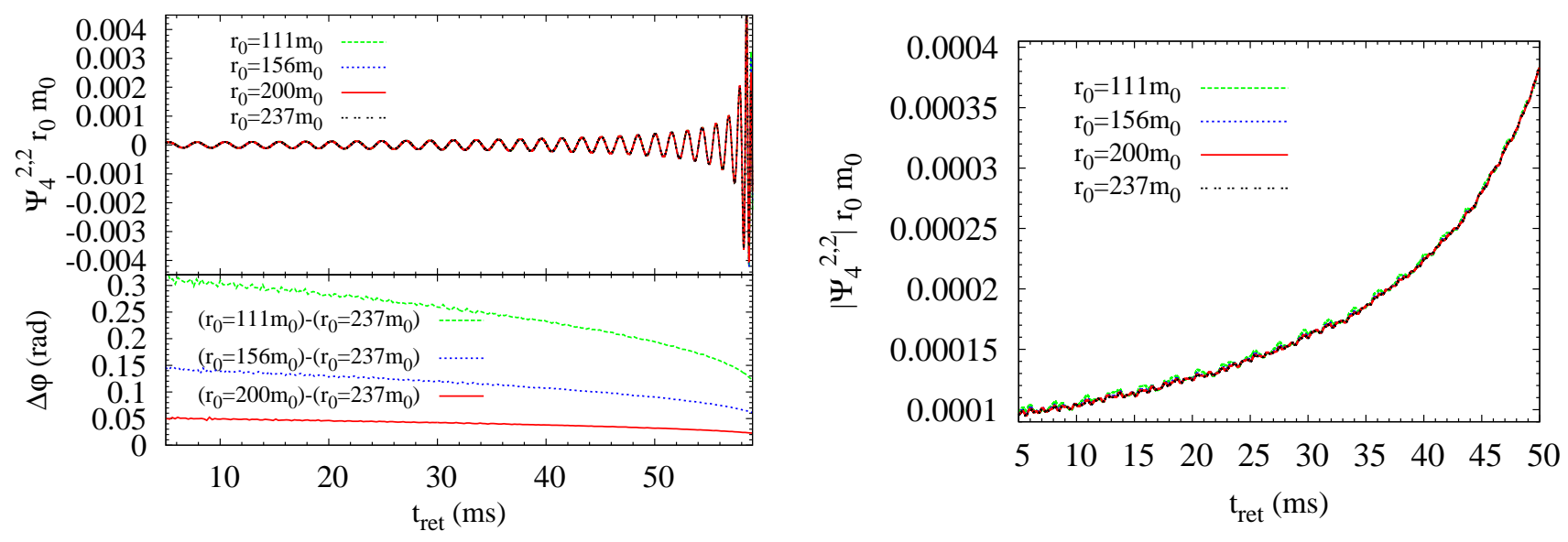

FIG. 1: The waveform (real part; left) and amplitude (right) of $\Psi_{4}^{2,2, \infty}\left(r_{0}, t_{\text {ret }}\right)$ as functions of $t_{\text {ret }}$ for several values of $r_{0}$ for the run with H4 EOS and the best grid resolution $(N=72)$. The lower plot of the left panel shows the phase differences of $\Psi_{4}^{2,2, \infty}\left(r_{0}\right)$ relative to $\Psi_{4}^{2,2, \infty}\left(r_{0}=237 m_{0}\right)$.

written as

$$
h^{l, m}\left(t_{\mathrm{ret}}\right)=\int d \omega \frac{\Psi_{4}^{l, m, \infty}(\omega)}{\max \left(\omega, \omega_{\mathrm{cut}}\right)^{2}} \exp \left(i \omega t_{\mathrm{ret}}\right),
$$

where $\Psi_{4}^{l, m, \infty}(\omega)$ is the Fourier transform of $\Psi_{4}^{l, m, \infty}\left(t_{\text {ret }}\right)$ and $\omega_{\text {cut }}$ is chosen to be $1.6 \Omega_{0}$. (Note that at the initial stage, the value of $\omega$ is $\left.2 \Omega_{0}>\omega_{\text {cut }}\right)$. We recall again that in this paper we pay attention only to $l=|m|=2$ modes because these are the dominant modes in particular for the equal-mass binaries. Then, from Eq. (3.3), we determine the evolution of the amplitude, i.e., $A^{l, m}=\left|h^{l, m}\right|$ as a function of $t_{\text {ret }}$.

Using Eq. (3.3), we can also define the evolution of the angular frequency as

$$
\omega\left(t_{\text {ret }}\right):=\frac{\left|\dot{h}^{2,2}\right|}{\left|h^{2,2}\right|},
$$

and then, the evolution of the gravitational-wave phase is calculated by

$$
\Phi\left(t_{\mathrm{ret}}\right):=\int^{t_{\mathrm{ret}}} d t^{\prime} \omega\left(t^{\prime}\right) .
$$

Now, using $A^{2,2}$ and $\Phi$, the quadrupole gravitational waveform can be written by

$$
h^{2,2}\left(t_{\mathrm{ret}}\right)=A^{2,2}\left(t_{\mathrm{ret}}\right) \exp \left[i \Phi\left(t_{\mathrm{ret}}\right)\right] .
$$

Figure 2 plots the resulting gravitational waveforms and the evolution of $\Phi$ obtained in the simulations with different grid resolutions for the models with $\mathrm{H} 4$ (left) and APR4 EOS (right). The upper panels plot the gravitational waveforms and these show that the merger time is earlier for the poorer grid resolutions. The middle panels plot the integrated wave phases for the pure numerical results with no reprocessing. These show that the phase evolution is spuriously faster for the poorer grid resolutions. However, we already know that the merger time converges approximately at 4th-order. Taking into account this fact, we stretch the time axis for the gravitational waveform by an appropriate factor as $t \rightarrow \eta t$ where $\eta(>1)$ is the constant stretching factor. This factor should be larger for the results of the poorer grid resolutions. Here, this reprocessing is performed in the same manner as in [23]: $t_{\text {ret }}$ and $\Phi$ are modified as $t_{\text {ret }} \rightarrow \eta t_{\text {ret }}$ and $\Phi \rightarrow \eta \Phi$. We will show that the phase evolution matches very well among the waveforms with different grid resolutions after this scaling performed in terms of this single parameter $\eta$. Later, $\eta$ will be also used for determining the convergence order and for obtaining the resolution-extrapolated waveform.

As a first step for this stretching procedure, we have to determine the values of $\eta$. As the first substep, we carry out a procedure for finding the minimum value of the following integral

$$
\begin{aligned}
I=\min _{\eta^{\prime}, \phi} \int_{t_{i}}^{t_{f}} d t_{\mathrm{ret}} & \mid A_{2}^{2,2}\left(\eta^{\prime} t_{\mathrm{ret}}\right) \exp \left[i \eta^{\prime} \Phi_{2}\left(\eta^{\prime} t_{\mathrm{ret}}\right)+i \phi\right] \\
& -\left.A_{1}^{2,2}\left(t_{\mathrm{ret}}\right) \exp \left[i \Phi_{1}\left(t_{\mathrm{ret}}\right)\right]\right|^{2},
\end{aligned}
$$

where $A_{1}^{2,2}$ and $\Phi_{1}$ are, respectively, the amplitude and integrated phase of the gravitational waveform for the best-resolved run $(N=72)$ and $A_{2}^{2,2}$ and $\Phi_{2}$ are those for less-resolved runs. The free parameters, $\eta^{\prime}$ and $\phi$, are varied for a wide range and from 0 to $2 \pi$, respectively, to search for the possible minimum value of $I . t_{i}$ and $t_{f}$ are chosen to be $5 \mathrm{~ms}$ and $t_{\mathrm{mrg}}$ of the best resolved run, respectively. Here, the reason for choosing $t_{i}=5 \mathrm{~ms}$ is that for their early stage with $t_{\text {ret }} \lesssim 5 \mathrm{~ms}$, the numerical waveforms have a relatively large modulation in amplitude and phase due to junk radiation.

We find for our present simulation results that for the second-finest, third-finest, and poorest resolution runs, 

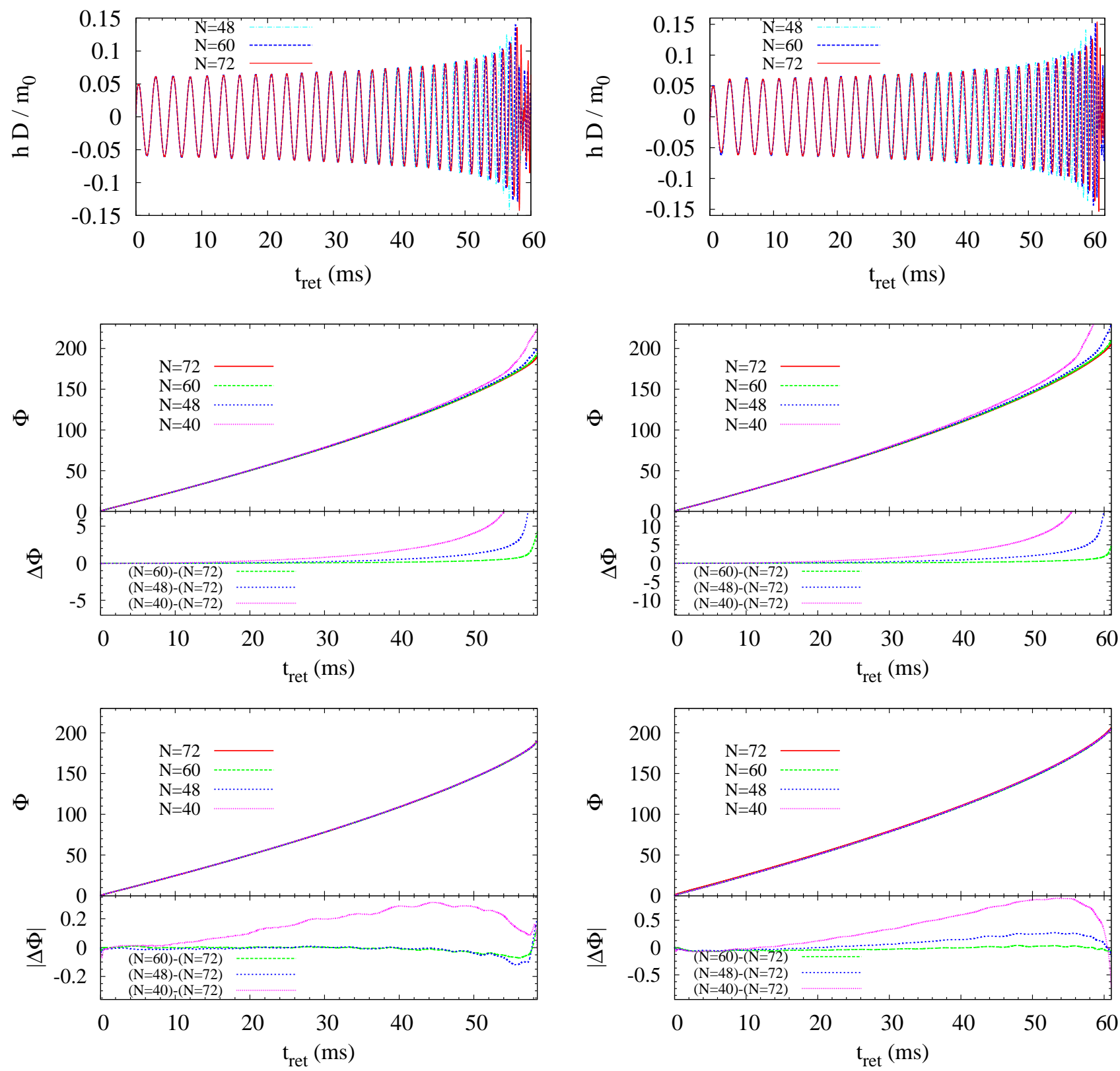

FIG. 2: The gravitational waveforms and the evolution of the gravitational-wave phase for four different grid-resolution runs with the H4 EOS (left three panels) and with the APR4 EOS (right three panels). $N$ indicates the grid resolution, $\Delta x_{8} \propto N^{-1}$. The upper panels show the gravitational waveforms for three different grid resolutions. The middle panels show the pure numerical wave phases and the bottom panels show the results obtained after the stretching of time and phase according to the convergence property (for $N=40,48$, and 60 ). The lower plots in middle and bottom panels show the phase disagreement between the purely numerical wave phase for $N=72$ and the lower-resolution results. Note that for $N=72, t_{\mathrm{mrg}}=58.43(\mathrm{~ms})$ for the $\mathrm{H} 4 \mathrm{EOS}$ and $t_{\mathrm{mrg}}=61.08(\mathrm{~ms})$ for the APR4 EOS.

$\eta^{\prime}=1.00646,1.02241$, and 1.06000 for the H4 EOS and $\eta^{\prime}=1.00650,1.02931$, and 1.09118 for the APR4 EOS. The mismatched factors, respectively, are $I / I_{0}=7.4 \times$ $10^{-6}, 2.3 \times 10^{-5}$, and $1.4 \times 10^{-4}$ for the H4 EOS and $I / I_{0}=7.4 \times 10^{-6}, 1.1 \times 10^{-4}$, and $1.4 \times 10^{-3}$ for the
APR4 EOS. Here, we define

$$
I_{0}:=\int_{t_{i}}^{t_{f}} d t_{\mathrm{ret}}\left|A_{1}^{2,2}\left(t_{\mathrm{ret}}\right)\right|^{2}
$$

The cross correlation of two waveforms is approximately estimated as $1-\sqrt{I / 2 I_{0}}$. This implies that the cross correlation between the waveforms of the best-resolved 
run and reprocessed less-resolved runs are approximately $99.9 \%, 99.8 \%, 99.4 \%$ for the H4 EOS and $99.9 \%, 99.5 \%$, and $98.2 \%$, respectively. This shows that the accuracy is not very good in the low-resolution runs for the APR4 EOS, for which the compactness is larger than that for the $\mathrm{H} 4 \mathrm{EOS}$, and hence, a finer grid resolution would be necessary for a well-resolved simulation. For both EOS, the reprocessed waveforms in the poorest-resolution run are found to be not very accurate, and hence, in the following, we will perform a convergence study employing the waveforms of the first-, second-, third-resolved runs (labeled by $N=72,60$, and 48 , respectively).

The bottom panels of Fig. 2 show the results obtained for this time-stretching procedure. It is found that four curves of $\Phi$ originally with different grid resolutions approximately overlap with each other. In particular, the degree of the overlapping is quite good between the finest and second-finest runs (see the difference of the integrated phase shown in the lower plot of the bottom panels of Fig. 21): For both EOS, the disagreement of $\Phi$ for these reprocessed data is much smaller than 0.1 radian except for the final moment of the last orbits, at which the disagreement steeply increases: however it is at most $\sim 0.2$ radian. This suggests that the time stretching method can be used for obtaining the extrapolated waveform for $\Delta x_{8} \rightarrow 0$ if we accept the error of the integrated phase up to $\sim 0.2$ radian.

We next try to obtain an extrapolated waveform for $\Delta x_{8} \rightarrow 0$ by using the time stretching method for the well resolved models. For this procedure, we have to determine the order of the convergence appropriately. In the above, we found that the numerical waveform in the poorest run is not very reliable even after the reprocessing. Thus, we determine the order of the convergence from the three better-resolved runs. (Note that if we employ the poorest-resolved waveforms for determining it, the order of the convergence is spuriously overestimated.) Using the values of $\eta^{\prime}-1$, the order of the convergence, $p$, is determined from

$$
\frac{(72 / 48)^{p}-1}{(72 / 60)^{p}-1}=\left\{\begin{array}{l}
\frac{0.02241}{0.00646} \text { for } \mathrm{H} 4 \\
\frac{0.02931}{0.00650} \text { for APR4 }
\end{array}\right.
$$

which give $p \approx 3.42$ and 5.10 , for the H4 and APR4 EOS, respectively. This indicates that the stretching factor for the best-resolved run to reproduce the limiting waveform with $\Delta x_{8} \rightarrow 0$ is $\eta \approx 1.00746$ and 1.00424 for the $\mathrm{H} 4$ and APR4 EOS, respectively. This implies that for these models, the exact merger time would be $t_{\mathrm{mrg}} \approx 58.87 \mathrm{~ms}$ and $61.34 \mathrm{~ms}$, respectively, whereas they were $58.43 \mathrm{~ms}$ and $61.08 \mathrm{~ms}$ for the best-resolved run. Namely, the error in the merger time is still much larger than $0.1 \mathrm{~ms}$ even for the best-resolved run: For obtaining the waveforms of the error in the merger time smaller than $0.1 \mathrm{~ms}$, a simulation with $N \gtrsim 100$ would be necessary. We note that if we extrapolate the value of $\eta$ for the best-resolved runs assuming the third- and fourth-order convergences of $\eta^{\prime}$, the value of $\eta$ becomes, respectively, 1.00887 and 1.00601 for the H4 EOS and 1.00893 and 1.00605 for the APR4 EOS. For the hypothetical fourth-order convergence, the predicted merger time would be $t_{\mathrm{mrg}}=58.78 \mathrm{~ms}$ for the H4 EOS and $61.45 \mathrm{~ms}$ for the APR4 EOS. Thus, it is safe to keep in mind that the extrapolated merger time still has an error of $\sim 0.1 \mathrm{~ms}$ due to the uncertainty in $p$. Since the merger time is $\sim 60 \mathrm{~ms}$ and total gravitationalwave phase is $\sim 200$ radian for both EOS, we should keep in mind the phase error of $200 \times(0.1 / 60) \sim 0.3$ radian.

\section{COMPARISON BETWEEN NUMERICAL-RELATIVITY AND EFFECTIVE-ONE-BODY WAVEFORMS}

Figure 3 plots the extrapolated gravitational waveforms, the associated frequency, and the integrated gravitational-wave phase. For comparison, we plot the results by an effective-one-body (EOB) approach [13, 21, 45. (see appendix A for the EOB formalism that we employ in this work). To align the time and phase of the numerical and EOB waveforms, we first calculate a correlation like Eq. (3.7) for $5 \mathrm{~ms} \leq t_{\text {ret }} \leq 20 \mathrm{~ms}$ between the numerical and EOB waveforms, varying the time and phase of the EOB waveform. These parameters are determined by searching for the set of the values that give the minimum of this integral.

Figure 3 shows that up to $f \sim 700 \mathrm{~Hz}$ (at $t_{\text {ret }} \approx 54 \mathrm{~ms}$ ), the EOB result well reproduces the extrapolated waveforms for both $\mathrm{H} 4$ and APR4 EOS: In particular for the APR4 EOS for which the compactness is large and the tidal deformability is small, the agreement is quite good. For both EOS, the error in the frequency is smaller than $1 \%$ and the phase error is smaller than 0.1 radian for $f \lesssim 700 \mathrm{~Hz}$ (with $t_{\text {ret }} \geq 5 \mathrm{~ms}$ ). However, for the last a few cycles, the agreement between the extrapolated and EOB waveforms becomes poor. Here, note that this disagreement cannot be explained by the error in the numerical waveform, because we have already estimated that the phase error in the numerical waveform would be smaller than $\sim 0.3$ radian. The magnitude of the error is larger for the H4 EOS. The possible reason for this disagreement is that in the current version of the EOB formalism, the tidal effects are not fully taken into account (e.g., non-linear tidal effects and non-stationary effects are not included). Namely, if the degree of the tidal deformation becomes high, the approximation could be poor.

In the final inspiraling stage for the model with the $\mathrm{H} 4$ EOS, the neutron stars are significantly deformed, and the attractive force associated with the tidal deformation is enhanced: The relative fraction of the approaching velocity induced by the tidal effect to that by other general relativistic effects such as gravitational-radiation reaction is larger for the binary of larger-radius neutron stars. The missing tidal effects could give a significant damage in the current version of the EOB formalism. By 

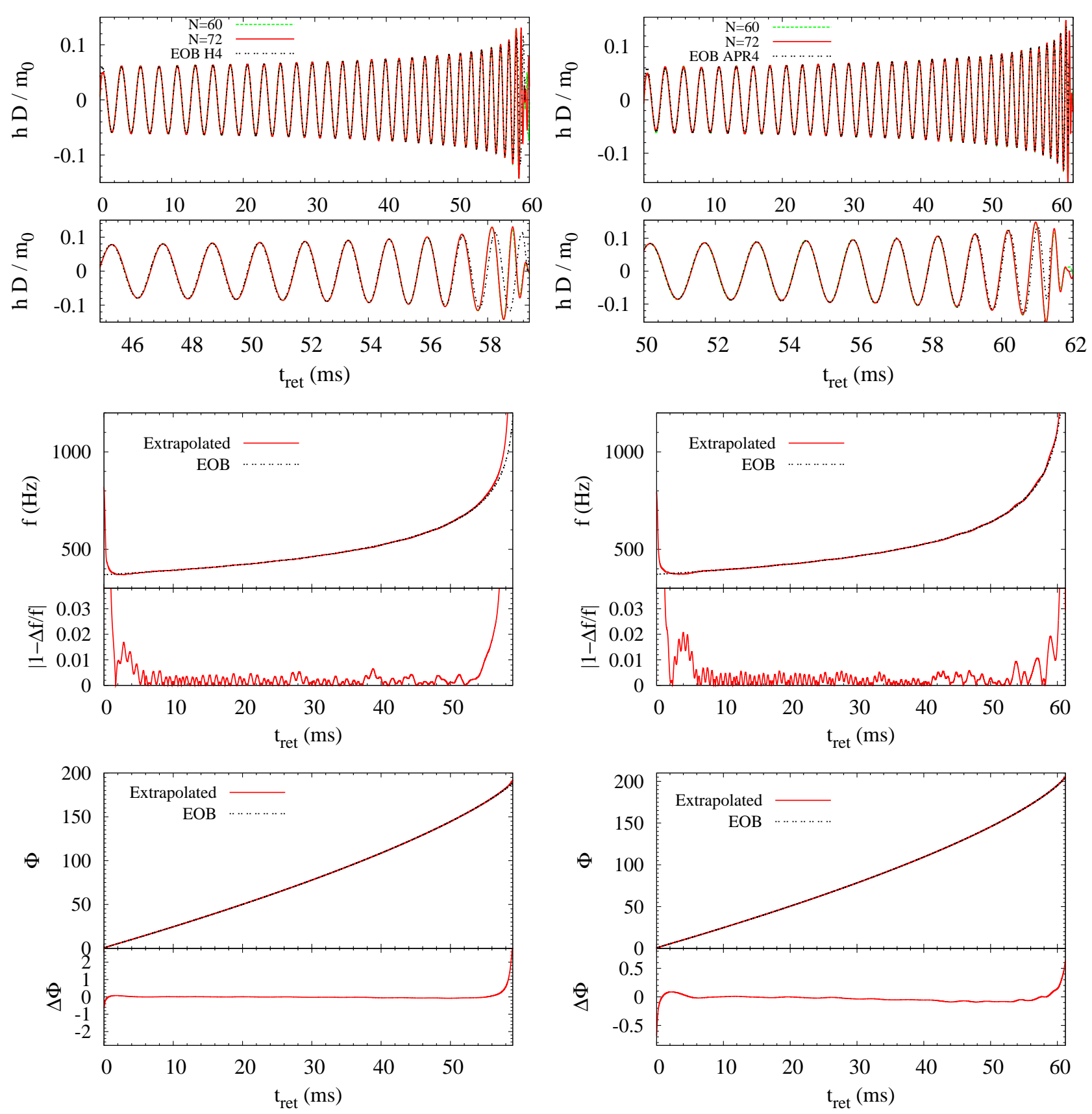

FIG. 3: The extrapolated gravitational waveform and related quantities for the models with the H4 (left) and APR4 EOS (right). Top: The extrapolated waveforms for the best-resolved $(N=72)$ and second-best-resolved $(N=60)$ runs are plotted (two waveforms overlap quite well with each other and we cannot distinguish them in the figure). The waveform by an effectiveone-body calculation is plotted together. The lower panels focus on the late inspiral waveforms. Middle: The extrapolated gravitational-wave frequency. In the lower panel of this, the absolute difference between the extrapolated result (with $N=72$ ) and EOB result is shown. Bottom: The extrapolated gravitational-wave phase. In the lower panel of this, the difference between the extrapolated result and EOB result is shown. We aligned the phases of the extrapolated and EOB waveforms at $t_{\mathrm{ret}}=5 \mathrm{~ms}$.

contrast, for the model with the APR4 EOS, the agreement between the extrapolated and EOB waveforms is quite good even at the last orbit. The total phase error is smaller than $\sim 0.7$ radian, which is comparable to that in the error associated with the uncertainty of the extrap- olation. This implies that for the binary of small-radius neutron stars, the current version of the EOB formalism would be already robust if we accept the phase error of $\sim 1$ radian (see also Ref. [20]).

The missing tidal effects in the EOB formalism cannot 


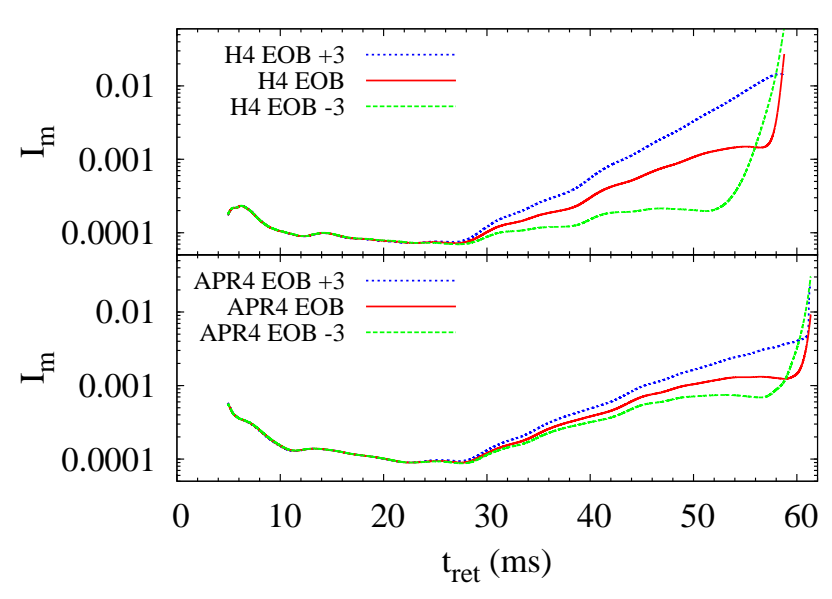

FIG. 4: The evolution of the mismatch, $I_{m}\left(t_{\text {ret }}\right)$, between the extrapolated waveform and $\mathrm{EOB}$ waveforms. "EOB \pm 3 " denotes that the EOB waveforms are employed artificially increasing or decreasing the neutron-star compactness by $3 \%$.

be compensated even if we artificially modify the value of the tidal deformability (or compactness). Figure 4 plots the evolution for the degree of the mismatch between the extrapolated waveform and the EOB waveform. For comparison, we calculated the mismatch employing the EOB waveforms in which the compactness of neutron stars is varied by $\pm 3 \%$. Here, the mismatch is defined by

$$
I_{m}\left(t_{\mathrm{ret}}\right):=\frac{1}{2} \frac{\left(h-h_{\mathrm{eob}} \mid h-h_{\mathrm{eob}}\right)}{\left(h_{\mathrm{eob}} \mid h_{\mathrm{eob}}\right)},
$$

where

$$
\left(h_{1} \mid h_{2}\right):=\int_{t_{i}}^{t_{\mathrm{ret}}} h_{1}\left(t_{\mathrm{ret}}^{\prime}\right) h_{2}^{*}\left(t_{\mathrm{ret}}^{\prime}\right) d t_{\mathrm{ret}}^{\prime}
$$

Again, $t_{i}$ is chosen to be $5 \mathrm{~ms}$. Here, $h$ and $h_{\text {eob }}$ denote an extrapolated waveform and a waveform by the EOB formalism, respectively. We note that the following relation is approximately satisfied for small values of $I_{m}\left(t_{\text {ret }}\right)$ :

$$
1-I_{m}\left(t_{\mathrm{ret}}\right) \approx \frac{\left(h \mid h_{\mathrm{eob}}\right)}{\sqrt{(h \mid h)\left(h_{\mathrm{eob}} \mid h_{\mathrm{eob}}\right)}} .
$$

From Fig. 4, we first reconfirm that the degree of the mismatch is steeply increased for the last inspiral orbit. This indicates that the tidal effect would not be sufficiently taken into account in the current version of the $\mathrm{EOB}$ formalism, although for other inspiral orbits, the performance of the EOB formalism appears to be quite good. It is also found that the extrapolated waveforms cannot be accurately reproduced even if we simply change the tidal deformability: If its value is artificially increased, the phase evolution is accelerated, and as a result, the mismatch is increased in an earlier inspiral stage. If it is artificially decreased, the merger is delayed, and as a result, the mismatch is badly increased near the last orbit.
This suggests that a tidal effect, which is not included, should be taken into account for improving the performance of the EOB formalism.

\section{SUMMARY}

We presented our latest numerical results of longterm simulations for the inspiraling binary neutron stars of equal mass. By a careful resolution study and extrapolation procedure, we obtain an accurate waveform: The estimated total phase error is smaller than $\sim 0.3$ radian for the total integrated phase of $\sim 200$ radian and the maximum error in the wave amplitude is smaller than $3 \%$. Using these accurate waveforms, we calibrated the waveforms derived by the latest EOB formalism. We show that for a binary of compact neutron stars (with their radius $11.1 \mathrm{~km}$ ), the waveform by the EOB formalism agrees quite well with the numerical waveform so that the total phase error is smaller than 1 radian. By contrast, for a binary of less compact neutron stars (with their radius $13.6 \mathrm{~km}$ ), the EOB and numerical waveforms disagree with each other in the last a few wave cycles, resulting in the total phase error of $\sim 3$ radian. We infer that this is due to the missing of some tidal effect such as nonlinear tidal effect in the current version of the EOB formalism, which should be taken into account for improving its performance.

In this work, we employed only two representative EOS and a binary of particular mass. For systematically improving the EOB formalism, we have to derive waveforms of wider sets of EOS and binary mass. We plan to perform more simulations in the future work and to present a larger number of the waveforms using the prescription developed in this paper.

\section{Acknowledgments}

This work was supported by Grant-in-Aid for Scientific Research (24244028) of Japanese MEXT. KK is supported by JSPS Postdoctoral Fellowship for Research Abroad.

\section{Appendix A: Effective one body formalism}

In this work, we employ an EOB formalism for inspiraling binary neutron stars, which is described in Ref. [20]. The base point-particle dynamics for this EOB formalism is calibrated by the latest binary-black-hole merger simulations [46] and the tidal effects are taken into account based on the prescription of Refs. [13, 19, 21]. Here, we briefly review this type of the EOB formalisms and describe our choice.

We consider a binary system composed of stars $A$ and $B$ with mass of $M_{A}$ and $M_{B}$. The EOB effective metric 
is defined by

$$
d s_{\mathrm{eff}}^{2}=-A(r) d t^{2}+\frac{D(r)}{A(r)} d r^{2}+r^{2}\left(d \theta^{2}+\sin ^{2} \theta d \phi^{2}\right),
$$

where $(r, \phi)$ are dimensionless coordinates and their canonical momenta are $\left(p_{r}, p_{\phi}\right)$. We replace the radial canonical momentum $p_{r}$ with the canonical momentum $p_{r_{*}}$, where a tortoise-like radial coordinate $r_{*}$ is given by

$$
\frac{d r_{*}}{d r}=\frac{\sqrt{D(r)}}{A(r)} .
$$

Then, the binary dynamics can be described by the EOB Hamiltonian

$$
H_{\text {real }}\left(r, p_{r_{*}}, p_{\phi}\right)=M c^{2} \sqrt{1+2 \nu\left(\hat{H}_{\mathrm{eff}}-1\right)},
$$

where $\nu:=M_{A} M_{B} / M^{2}, M:=M_{A}+M_{B}$, and the effective Hamiltonian is defined by

$$
\hat{H}_{\mathrm{eff}}=\sqrt{p_{r_{*}}^{2}+A(r)\left(1+\frac{p_{\phi}^{2}}{r^{2}}+2(4-3 \nu) \nu \frac{p_{r_{*}}^{4}}{r^{2}}\right)} .
$$

The potential $A(r)$ is decomposed into two parts as

$$
A(r)=A_{\mathrm{pp}}(r)+A_{\text {tidal }}(r),
$$

where $A_{\mathrm{pp}}(r)$ is the point-particle potential and $A_{\text {tidal }}(r)$ is the term associated with tidal effects. The pointparticle potential including up to the fifth $\mathrm{PN}$ terms is

$$
\begin{aligned}
A_{\mathrm{pp}}(r)=P_{5}^{1} & {\left[1-2 u+2 \nu u^{3}+\nu a_{4} u^{4}\right.} \\
& +\nu\left(a_{5}^{\mathrm{c}}(\nu)+a_{5}^{\ln } \ln u\right) u^{5} \\
& \left.+\left(a_{6}^{\mathrm{c}}(\nu)+\nu a_{6}^{\ln }(\nu) \ln u\right) u^{6}\right],
\end{aligned}
$$

where $u:=1 / r$, and $P_{5}^{1}$ denotes a $(1,5)$ Padé approximant. Here, the following coefficients are analytically known [4, 48]

$$
\begin{aligned}
a_{4}= & \frac{94}{3}-\frac{41}{32} \pi^{2} \\
a_{5}^{\mathrm{c}}(\nu)= & -\frac{4237}{60}+\frac{2275}{512} \pi^{2}+\frac{256}{5} \ln 2+\frac{128}{5} \gamma \\
& +\left(-\frac{211}{6}+\frac{41}{32} \pi^{2}\right) \nu \\
a_{5}^{\ln }= & \frac{64}{5} \\
a_{6}^{\ln }(\nu)= & -\frac{7004}{105}-\frac{144}{5} \nu
\end{aligned}
$$

where $\gamma=0.5772156 \ldots$ is the Euler constant. Following Ref. [20], we take the effective form of $a_{6}^{\mathrm{c}}(\nu)$, with which results of binary-black-hole-merger simulations are reproduced accurately, as

$$
a_{6}^{\mathrm{c}}(\nu)=3097.3 \nu^{2}-1330.6 \nu+81.38 .
$$

The contribution of tidal effects to the potential is written as

$$
A_{\text {tidal }}(r)=-\sum_{l \geq 2}\left(\kappa_{l}^{A} u^{2 l+2} \hat{A}_{A}^{(l)}(u)+(A \leftrightarrow B)\right),
$$

where $\hat{A}_{A}^{(l)}$ includes the PN tidal effects and $\kappa_{l}^{A}$ is the tidal coefficients. Here, the subscripts $A$ and $B$ denote the stars $A$ and $B$. In this work, we include the tidal effects up to $l=4$. The coefficient $\kappa_{l}^{A}$ is related to the electric tidal Love number $k_{l}$ and the compactness $C$ as (see Table $\square$ for these values of the neutron stars studied in this work)

$$
\kappa_{l}^{A}=2 \frac{M_{B} M_{A}^{2 l}}{M^{2 l+1}} \frac{k_{l}^{A}}{C_{A}^{2 l+1}} .
$$

The tidal potential up to the next-to-next-to-leading corrections is

$$
\hat{A}_{A}^{(l)}(u)=1+\alpha_{A, 1}^{(l)} u+\alpha_{A, 2}^{(l)} u^{2} .
$$

The coefficients are analytically known as 13 .

$$
\begin{aligned}
\alpha_{A, 1}^{(2)} & =\frac{5}{2} X_{A}, \\
\alpha_{A, 2}^{(2)} & =\frac{337}{28} X_{A}^{2}+\frac{1}{8} X_{A}+3, \\
\alpha_{A, 1}^{(3)} & =\frac{15}{2} X_{A}-2, \\
\alpha_{A, 2}^{(3)} & =\frac{110}{3} X_{A}^{2}-\frac{311}{24} X_{A}+\frac{8}{3},
\end{aligned}
$$

where $X_{A}:=M_{A} / M$.

Recently, the tidal EOB was improved using resummation techniques [19]. We use the gravitational-self-force informed $l=2$ tidal potential as

$$
\begin{aligned}
\hat{A}_{A}^{(2)}(u)= & 1+\frac{3 u^{2}}{1-r_{\mathrm{LR}} u}+X_{A} \frac{\tilde{A}_{A}^{(2) 1 \mathrm{SF}}(u)}{\left(1-r_{\mathrm{LR}}\right)^{7 / 2}} \\
& +X_{A}^{2} \frac{\tilde{A}_{A}^{(2) 2 \mathrm{SF}}(u)}{\left(1-r_{\mathrm{LR}}\right)^{p}}
\end{aligned}
$$

where $p$ is an unknown parameter in the range of $4 \leq$ $p<6$ and we set $p$ to be $4 . r_{\mathrm{LR}}$ is the light-ring orbit. The forms of $\tilde{A}_{A}^{(2) 1 \mathrm{SF}}$ and $\tilde{A}_{A}^{(2) 2 \mathrm{SF}}$ are

$$
\begin{aligned}
\tilde{A}_{A}^{(2) 1 \mathrm{SF}}(u) & =\frac{5}{2} u\left(1-a_{1} u\right)\left(1-a_{2} u\right) \frac{1+n_{1} u}{1+d_{2} u^{2}}, \\
\tilde{A}_{A}^{(2) 2 \mathrm{SF}}(u) & =\frac{337}{28} u^{2}
\end{aligned}
$$

where the numerical coefficients $\left(a_{1}, a_{2}, n_{1}, d_{2}\right)$ are found in Ref. [19]. As in Ref. [20], we use the tidally corrected light ring orbit instead of $r_{\mathrm{LR}}=3$. For determining the value of $r_{\mathrm{LR}}$, we solve the following equation numerically

$$
A\left(u_{\mathrm{LR}}\right)+\left.\frac{1}{2} u_{\mathrm{LR}} \frac{d A}{d u}\right|_{u_{\mathrm{LR}}}=0,
$$


where the tidal part of the potential is included as Eq. (A14) and the value of $r_{\mathrm{LR}}$ for the binary neutron star models employed in this work is shown in Table I. Finally, the potential $D(u ; \nu)$ is given by

$$
D(u ; \nu)=\frac{1}{1+6 \nu u^{2}+2(23-3 \nu) \nu u^{3}} .
$$

For calculating the dynamics of the binary orbit under the potentials described above, we solve the EOB Hamilton's equations

$$
\begin{aligned}
\frac{d r}{d t} & =\frac{A(r)}{\sqrt{D(r)}} \frac{\partial H_{\text {real }}}{\partial p_{r_{*}}}, \\
\frac{d \phi}{d t} & =\frac{\partial H_{\text {real }}}{\partial p_{\phi}} \\
\frac{d p_{r_{*}}}{d t} & =-\frac{A(r)}{\sqrt{D(r)}} \frac{\partial H_{\text {real }}}{\partial r}, \\
\frac{d p_{\phi}}{d t} & =\mathcal{F}_{\phi} .
\end{aligned}
$$

Note that we do not include the radial part of the radiation-reaction force in Eq. [A26 20] because we find this choice advantageous for fitting the extrapolated gravitational waveforms. $\mathcal{F}_{\phi}$ is the radiation-reaction force given by

$$
\mathcal{F}_{\phi}=-\frac{1}{8 \pi \nu \omega} \sum_{l=2}^{8} \sum_{m=1}^{l}(m \omega)^{2}\left|R h_{l m}\right|^{2}
$$

where $\omega=d \phi / d t$ and $h_{l m}$ denotes the multipolar waveforms. Here, $h_{l m}$ is written as

$$
h_{l m}=h_{l m}^{0}+h_{l m}^{\mathrm{tidal}, \mathrm{A}}+h_{l m}^{\mathrm{tidal}, \mathrm{B}}
$$

where $h_{l m}^{0}$ includes the inspiral and plunge waveforms given in Ref. [46], and $h_{l m}^{\text {tidal,A }}$ and $h_{l m}^{\text {tidal,B }}$ are the tidal contributions due to the stars $\mathrm{A}$ and $\mathrm{B}$. They are given by Eqs. (A14)-(A17) of Ref. [21].
[1] J. Abadie et al. Nucl. Instrum. Meth. A 624, 223 (2010): T. Accadia et al. Class. Quant. Grav. 28, 025005 (2011) [Erratum-ibid. 28, 079501 (2011)]: K. Kuroda, Class. Quant. Grav. 27, 084004 (2010).

[2] R. O'Shaughnessy, V. Kalogera, and K. Belczynski, Astrophys. J. 716, 615 (2010).

[3] J. Abadie et. al., Class. Quantum Grav. 27, 173001 (2010).

[4] Adcanced LIGO, http://www.advancedligo.mit.edu/

[5] Advanced VIRGO, http://www.cascina.virgo.infn.it/advirgo/

[6] K. Kuroda: LCGT collaboration, Class. Quantum Grav. 27, 084004 (2010).

[7] C. Cutler, and E. E. Flanagan, Phys. Rev. D 49, 2658 (1994).

[8] J. S. Read, L. Baiotti, J. D. E. Creighton, J. L. Friedman, B. Giacomazzo, K. Kyutoku, C. Markakis, L. Rezzolla, M. Shibata, and K. Taniguchi, Phys. Rev. D 88, 044042 (2013).

[9] A. Bauswein, N. Stergioulas, and H. -T. Janka Phys. Rev. D 90, 023002 (2014)

[10] K. Takami, L. Rezzolla, and L. Baiotti, Phys. Rev. Lett 113, 091104 (2014).

[11] D. Lai, F. A. Rasio, and S. L. Shapiro, Astrophys. J. 420, 811 (1994).

[12] E. E. Flanagan, and T. Hinderer, Phys. Rev. D 77, 021502(R) (2008).

[13] D. Bini, T. Damour, and G. Faye, Phys. Rev. D 85, 124034 (2012).

[14] T. Mora, and C. M. Will, Phys. Rev. D 69, 104021 (2004).

[15] T. Hinderer, B. D. Lackey, R. N. Lang, and J. S. Read, Phys. Rev. D 81, 123016 (2010).

[16] M. Favata, Phys. Rev. Lett. 112, 101101 (2014).

[17] K. Yagi and N. Yunes, Phys. Rev. D 89, 021303 (2014).

[18] L. Wade, J. D. E. Creighton, E. Ochsner, B. D. Lackey, B. F. Farr, T. B. Littenberg, and V. Raymond, Phys,
Rev. D 89, 103012 (2014).

[19] D. Bini and T. Damour, Phys. Rev. D 90, 124037 (2014).

[20] S. Bernuzzi, A. Nagar, T. Dietrich, and T. Damour, arXiv: 1412.4553 .

[21] T. Damour, A. Nagar, and L. Villain, Phys. Rev. D 85, 123007 (2012).

[22] P. B. Demorest, T. Pennucci, S. M. Ransom, M. S. E. Roberts, and J. W. T. Hessels, Nature 467, 1081 (2010): J. Antoniadis et al., Science, 340, 448 (2013).

[23] K. Hotokezaka, K. Kyutoku, and M. Shibata, Phys. Rev. D 87, 044001 (2013).

[24] L. Baiotti, T. Damour, B. Giacomazzo, A. Nagar, and L. Rezzolla, Phys. Rev. D 84, 024017 (2011).

[25] M. Thierfelder, S. Bernuzzi, and B. Brügmann, Phys. Rev. D 84,044012 (2011).

[26] S. Bernuzzi, M. Thierfelder, and B. Brügmann, Phys. Rev. D 85, 104030 (2012).

[27] S. Bernuzzi, A. Nagar, M. Thierfelder, and B. Brügmann, Phys. Rev. D 86, 044030 (2012).

[28] D. Radice, L. Rezzolla, and F. Galeazzi, Mon. Not. Roy. Soc. 437, L46 (2014).

[29] T. Yamamoto, M. Shibata, and K. Taniguchi, Phys. Rev. D 78, 064054 (2008).

[30] M. Shibata and T. Nakamura, Phys. Rev. D 52 , 5428(1995): T. W. Baumgarte and S. L. Shapiro, Phys. Rev. D 59, 024007(1998): M. Campanelli, C. O. Lousto, P. Marronetti, and Y. Zlochower, Phys. Rev. Lett. 96, 111101 (2006): J. G. Baker, J. Centrella, D.-I. Choi, M. Koppitz, and J. van Meter, Phys. Rev. Lett. 96, 111102 (2006).

[31] D. Hilditch, S. Bernuzzi, M. Thierfelder, Z. Cao, W. Tichy, and B. Brügmann, Phys. Rev. D 88, 084057 (2013).

[32] K. Kyutoku, M. Shibata, and K. Taniguchi, Phys. Rev. D 90, 064006 (2014).

[33] B. Brügmann, J. A. Gonzalez, M. Hannam, S. Husa, U. Sperhake, and W. Tichy, Phys. Rev. D 77, 024027 (2008). 
[34] A. Kurganov and E. Tadmor, J. Comput. Phys. 160, 241 (2000).

[35] LORENE webpage: http://www.lorene.obspm.fr/ .

[36] L. Bildsten, and C. Cutler, Astrophys. J. 400, 175 (1992).

[37] C. S. Kochanek, Astrophys. J. 398, 234 (1992).

[38] J. S. Read, B .D. Lackey, B. J. Owen, and J. L. Friedman, Phys. Rev. D 79, 124032 (2009).

[39] A. Akmal, V. R. Pandharipande, and D. G. Ravenhall, Phys. Rev. C 58, 1804 (1998).

[40] N. K. Glendenning and S. A. Moszkowski, Phys. Rev. Lett. 67, 2414 (1991).

[41] K. Hotokezaka, K. Kiuchi, K. Kyutoku, T. Muranushi, Y. -i. Sekiguchi, M. Shibata and K. Taniguchi,Phys. Rev. D 88, 044026 (2013).

[42] C. O. Lousto, H. Nakano, Y. Zlochower, and M. Cam- panelli, Phys. Rev. D 82, 104057 (2010).

[43] H. Nakano, arXiv: 1501.0289.

[44] C. Reisswig and D. Pollney, Class. Quantum Grav. 28, 195015 (2011).

[45] T. Damour and A. Nagar, Phys. Rev. D 81, 084016 (2010).

[46] T. Damour, A. Nagar, and S. Bernuzzi, Phys. Rev. D $\mathbf{8 7 ,} 084035$ (2013). The code is available at https://eobnew.ihes.fr .

[47] D. Bini and T. Damour, Phys. Rev. D 87, 121501(R) (2013).

[48] E. Barausse, A. Buonanno, and A. Le Tiec, Phys. Rev. D 85, 064010 (2012). 